\title{
Rotavirus encephalopathy with concomitant acute cerebellitis: report of a case and review of the literature
}

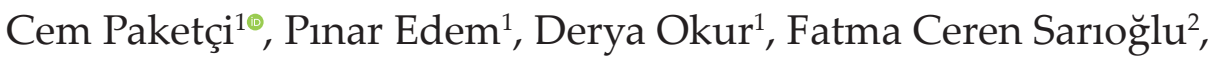 \\ Handan Güleryüz ${ }^{2}$, Erhan Bayram¹ ${ }^{1}$, Semra Hız Kurul ${ }^{1}$, Uluç Yişs ${ }^{1}$ \\ Divisions of ${ }^{1}$ Pediatric Neurology, Department of Pediatrics, ${ }^{2}$ Pediatric Radiology, Dokuz Eylül University Faculty of Medicine, İzmir, \\ Turkey.
}

\begin{abstract}
Rotavirus is a leading cause of gastroenteritis in children under 5 years of age. It is known that neurological manifestations like seizures, encephalopathy and encephalitis can rarely be seen due to rotavirus infections. Cerebellar involvement is extremely rare. We present an uncommon neurological manifestation of rotavirus infection in a 4-year-old Turkish child who presented with hypotonia, reduced consciousness and mutism. Magnetic resonance imaging revealed diffusion abnormalities in the splenium of corpus callosum and nucleus dentatus bilaterally. She was diagnosed with rotavirus cerebellitis. She improved well with dexamethasone and intravenous immunoglobulin but still has dysarthria and poor fine motor coordination.
\end{abstract}

Key words: central nervous system infection, encephalitis, encephalopathy, mutism, rotavirus.

Rotavirus is a common cause of gastroenteritis in children and may be complicated by central nervous system involvement. Afebrile seizures are common, but acute cerebellitis is a quite rare neurological complication. Cerebellar involvement has characteristic clinical and radiologic features. ${ }^{1}$ There is no established treatment for this condition, but some cases have been treated with intravenous immunoglobulin (IVIG) or steroids. We present a case of rotavirus associated acute cerebellitis treated with dexamethasone with a relatively favorable outcome.

\section{Case Report}

A four-year-old previously healthy girl was admitted to hospital with complaints of diarrhea, vomiting and fever. Intravenous hydration was administered with the diagnosis of acute gastroenteritis and dehydration.

\section{Cem Paketçi}

paketci@hotmail.com

Received 18th December 2018; revised 9th February 2019; accepted 9th March 2019
She was discharged with prescription of metoclopramide. Three days after discharge, she was readmitted to our hospital with altered mental status and inability to speak. Her neurologic symptoms started three days after diarrhea on the day of admission to our hospital. There was no history of infectious disease in the last month. She was fully vaccinated excluding rotavirus vaccine, which is not a routine component of vaccine schedule in Turkey. No vaccine has been administered in the last three months.

Neurological examination revealed a deterioration in the level of consciousness with a Glasgow Coma Scale score of 10 (E2, V3, M5) and generalized muscle weakness. Muscle strength was $2 / 5$ in upper and $3 / 5$ in lower limbs symmetrically. Deep tendon reflexes were normal. She could not speak and could not walk. Her cranial nerve examination was unremarkable. Cerebellar tests could not be done due to consciousness disturbance. Remainder of the physical examination was normal.

Laboratory data, including serum glucose, 
ammonia, lactate an electrolyte levels were normal except for an elevated C-reactive protein of $2.08 \mathrm{mg} / \mathrm{dl}(\mathrm{N}$ : $0.2-0.5 \mathrm{mg} / \mathrm{dl})$. Cranial computed tomography was normal.

Magnetic resonance imaging (MRI) of the brain showed a hyperintensity in the splenium of the corpus callosum (SCC) on T2-weighted and diffusion weighted images with corresponding diffusion restriction on Apparent Diffusion Coefficient (ADC) mapping (Fig. 1). Cerebrospinal fluid (CSF) examination revealed no cells, protein $51.6 \mathrm{mg} / \mathrm{dl}(\mathrm{N}$ : $<40$ $\mathrm{mg} / \mathrm{dl})$ and glucose $81 \mathrm{mg} / \mathrm{dl}(\mathrm{N}$ : $60-80 \mathrm{mg} /$ dl) with simultaneous blood glucose of 113 $\mathrm{mg} / \mathrm{dl}$. Empirical treatment with intravenous ceftriaxone, vancomycin and acyclovir were initiated. Rotavirus antigen was positive in stool specimen 3 days after the onset of diarrhea, on the first day of onset of neurologic symptoms at the admission to our hospital. Tentative diagnosis was rotavirus-associated
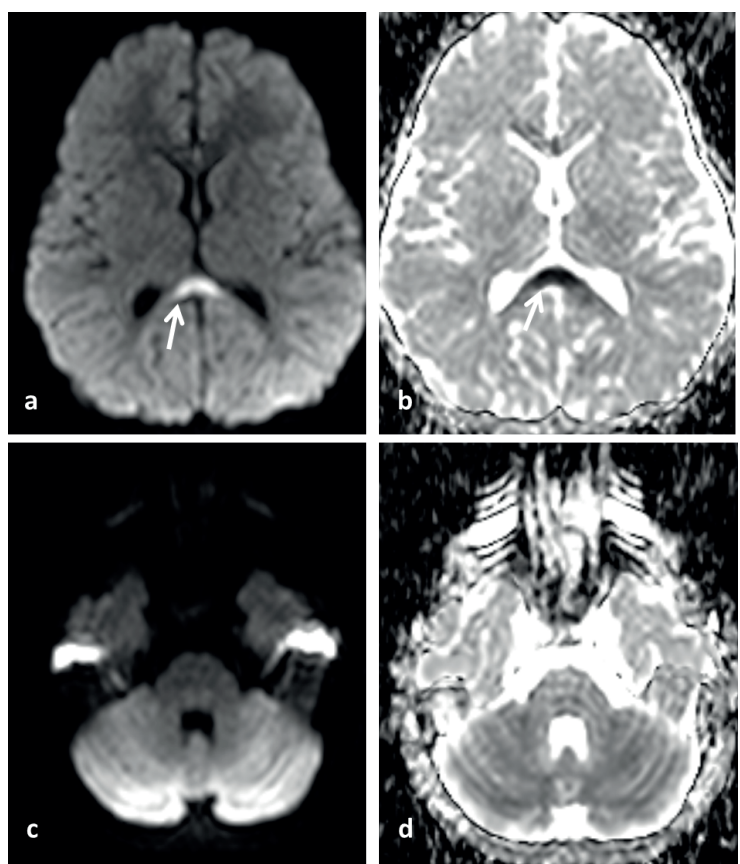

Fig. 1. Magnetic resonance (MR) imaging of patient on day-1. B 1000-weighted (a) and ADC (b) images showed that the diffusion restriction in the splenium of corpus callosum (arrows). B 1000-weighted (c) and ADC (d) images also showed no abnormalities in the cerebellum initially. mild encephalopathy with a reversible splenial lesion (MERS).

On day-2, she was still encephalopathic, mute and muscle strength were the same as admission. Electroencephalography was normal. Antimicrobial treatment was discontinued once CSF culture revealed no bacterial growth and PCR was negative for herpes simplex virus, enterovirus and adenovirus. Rotavirus could not be tested in CSF. Intravenous immunoglobulin was given for two days $(1 \mathrm{~g} /$ $\mathrm{kg}$ /day). On day-3, despite the improvement in consciousness, she was still mute which is a well-known feature of acute cerebellitis. Hence a control brain MRI was requested on suspicion of acute cerebellitis. This showed reduced diffusion in dentate nuclei. There was also a slight signal deviation on the T2-weighted sequence in the same area. Previous lesion at SCC disappeared (Fig. 2). After a diagnosis of acute cerebellitis, dexamethasone $(1 \mathrm{mg} / \mathrm{kg} /$ day $)$ was administered at third day of admission and continued for 5 days followed by dose tapering of 5 days. From the 5 th day of admission, our patient had improved consciousness, but she was still hypotonic and bedridden. Her movements were uncoordinated and unpredictable. Mutism became more prominent. She received physical therapy daily. After two weeks of illness, she was still mute, had head and neck control but could not sit without support.

Four months after onset, a follow-up brain MRI indicated marked cerebellar atrophy (Fig. 3). She was able to walk for a few steps with a wide-base gait and able to speak two or three word sentences. Her speech was still slow and dysarthric. She was able to walk but her fine motor coordination was poor. She still continues to improve, but the recovery is slow. An informed consent was received from the parents for publication.

\section{Discussion}

We report a case of rotavirus encephalopathy with concomitant acute cerebellitis. Although 

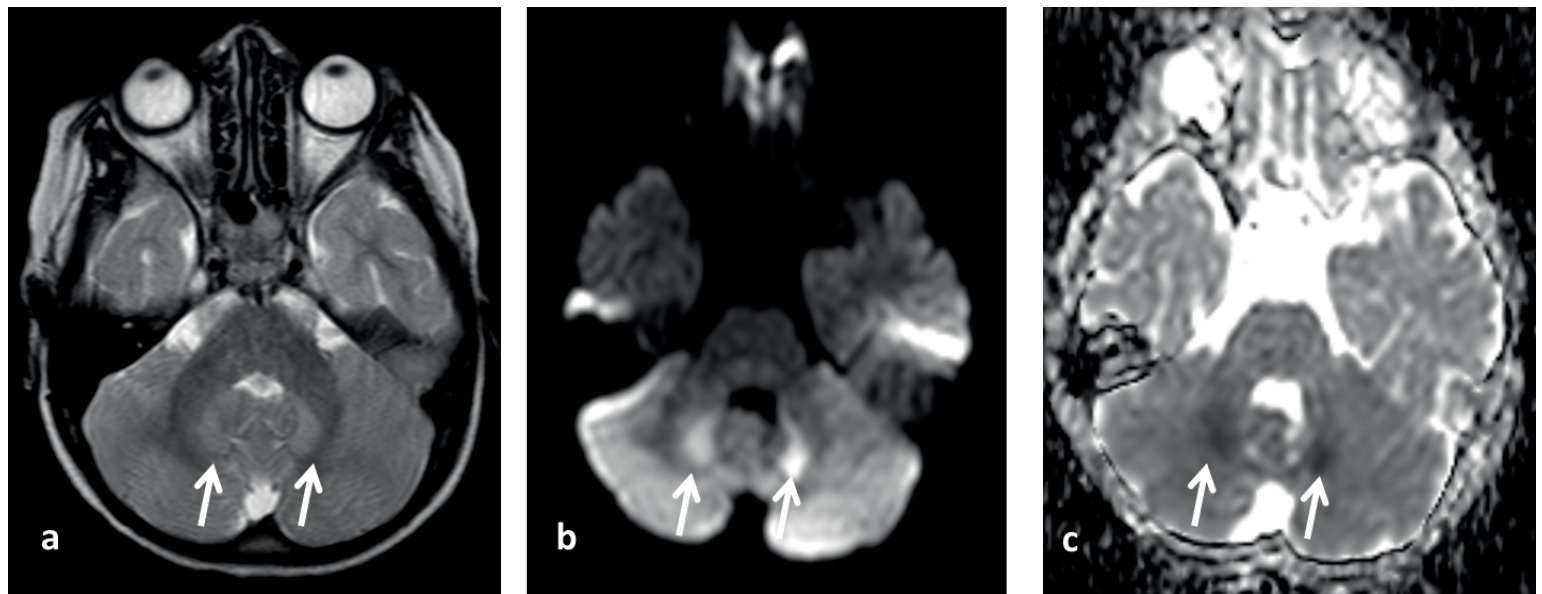

Fig. 2. Magnetic resonance (MR) imaging of patient on day-3. T2-weighted axial image (a) demonstrated that the high signal intensity in dentate nuclei (arrows). B 1000-weighted (b) and ADC (c) images also showed that the diffusion restriction in the same areas (arrows).
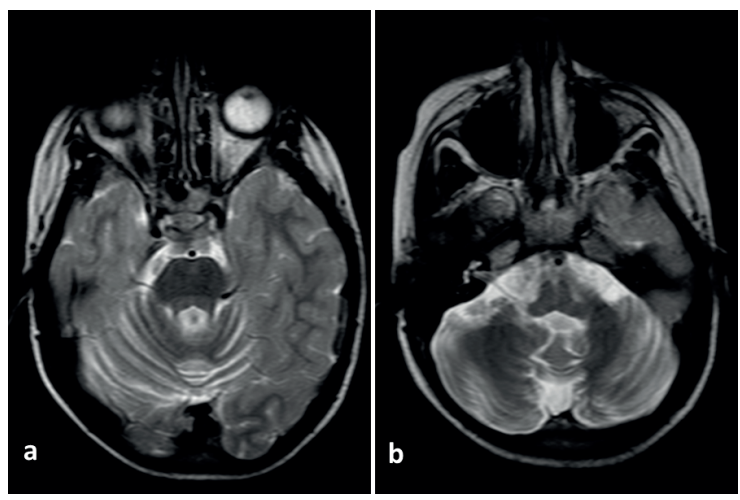

Fig. 3. Follow-up magnetic resonance (MR) imaging at four months. Diffuse cerebellar atrophy was seen in T2-weighted axial images (a-b).

rotavirus infection is common, neurological manifestations occur in $2 \%-5 \%$ of patients with rotavirus gastroenteritis. ${ }^{2}$ The pathogenesis of neurological signs of rotavirus infection is not clearly understood. Direct invasion of central nervous system, high radical activity of nitric oxide metabolites due to the damage of enterocytes by toxin like proteins such as the non-structural protein 4 and dissemination of the virus by the enteric nervous system are possible mechanisms. ${ }^{2,3}$

Cerebellitis associated with rotavirus infection has been described previously. However, there are only few patients in the literature. To date 23 cases with cerebellar involvement due to rotavirus infection have been reported (Table I). The clinical features of rotavirus associated encephalopathy and cerebellitis can vary widely. Consciousness disturbance, mutism, ataxia and hypotonia are the most characteristic findings. Seizures and intentional tremor are also frequent. Mutism is the most important and distinct finding of acute cerebellitis associated with rotavirus infection. ${ }^{1}$ Cerebellar mutism is generally a common complication of posterior fossa surgery in children. Non-surgical cerebellar mutism is rare, but can be seen after vascular events, trauma or inflammation. ${ }^{4}$ Although the underlying mechanism is unclear, it is suggested to result from impaired coordination of the articulatory muscles due to the damage of cerebello-thalamo-cerebral pathway. ${ }^{5}$ Cerebellar mutism was seen as an initial symptom in most patients with rotavirus cerebellitis, including our patient (19 of the 24 patients). Three patients suffered from dysarthtria. The mean duration of rotavirus associated cerebellar mutism is about a month as in our case. ${ }^{1}$

The typical MRI findings of rotavirus associated acute cerebellitis are a reversible splenial lesion in the early stages, abnormal signal intensity in the cerebellar white matter/nuclei and increased signal intensity in the cerebellar cortex respectively. The most likely finding in 


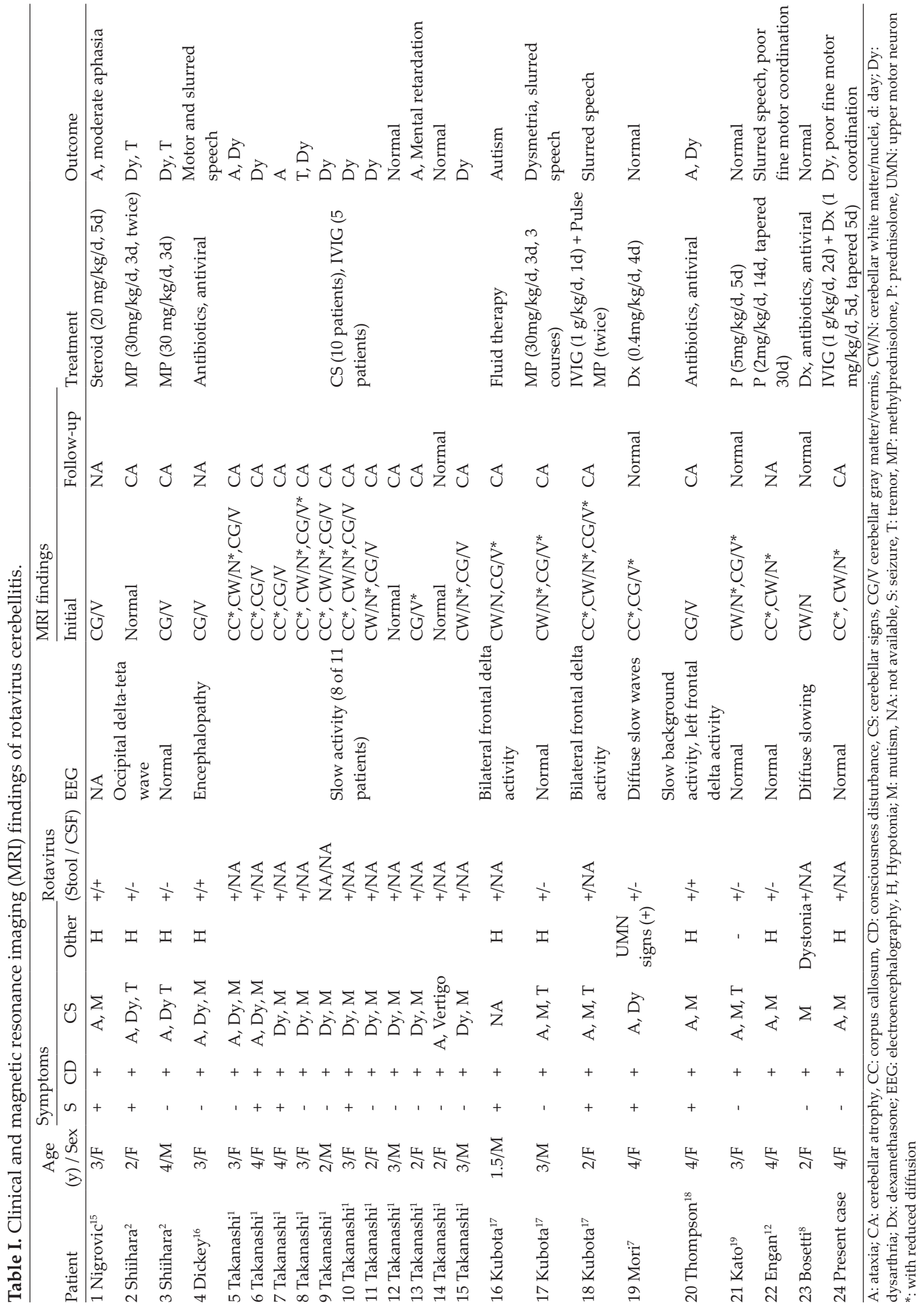


the long term follow-up is cerebellar atrophy. ${ }^{1}$ First MRI of our patient showed a T2-weighted hyperintensity and restricted diffusion in the SCC. Acute consciousness alteration after three days of fever, diarrhea and vomiting, demonstrates acute encephalopaty. Clinical findings and isolated MRI lesion with restricted diffusion in the SCC suggested rotavirusassociated MERS. But prominent mutism with hypotonia pointed cerebellar involvement. Second MRI on day 3 showed a T2-weighted hyperintensity with reduced diffusion in dentate nuclei. Restricted diffusion at SCC disappeared. These findings supported the diagnosis of acute cerebellitis and consistent with the literature.

Although somnolence, altered mental status and the loss of consciousness considered an encephalitis, lack of pleocytosis and negative culture results in the CSF suggested an encephalopathy rather than an encephalitis. Acute demyelinating encephalomyelitis (ADEM) often presents altered mental status, focal neurological signs and seizures which develop days to weeks after a viral infection or vaccination. Unlike our patient, mild CSF pleocytosis is expected and MRI lesions in ADEM, are typically asymmetrical, usually involve the white matter and resolve over months. ${ }^{6}$

Cerebellar atrophy is the most common finding on follow-up MRI (17 of the 24 patients). Three patients' follow-up MRI was not available/not done, four were normal. Speech disturbance is the most frequent long-term sequelae. All of the patients with speech disturbance as an outcome had cerebellar atrophy on follow-up MRI. Inversely there were no speech disturbance for four patients with cerebellar atrophy. Followup MRI of our patient at four months from onset showed cerebellar atrophy as in most patients.

No specific recommended treatment for rotavirus cerebellitis is available. Treatment with corticosteroids (methylprednisolone, prednisolone, dexamethasone) and IVIG has been used. Only two cases were treated with dexamethasone in the literature before and both showed complete recovery. One of these patients received dexamethasone therapy 0.4 $\mathrm{mg} / \mathrm{kg} /$ day for 4 days starting from day $5 .{ }^{7}$ The other patient was started on dexamethasone on day 2 , the dose was unknown. ${ }^{8}$ Both patients recovered completely without any sequelae. This patient was treated with IVIG and dexamethasone in an attempt to suppress an expected inflammatory process. She received infusions of $1 \mathrm{~g} / \mathrm{kg}$ IVIG for two days starting from day two and $1 \mathrm{mg} / \mathrm{kg} / \mathrm{g}$ dexamethasone for five days starting from day three. Our patient has mild dysarthria and poor fine motor coordination after four months but she still continues to improve. Dexamethasone may be a good choice in the treatment of patients with acute cerebellitis and may be preferred due to its strong anti-inflammatory effects.

The impact of rotavirus vaccination on seizures and other neurological complications is still not clear and research results are contradictory. ${ }^{9,10}$ Although the majority of studies are focused on seizures, it has been shown that the vaccination may be protective against severe complications of rotavirus. ${ }^{11}$ Only one of the patients in the literature had information about vaccination who was not vaccinated like our patient. ${ }^{12}$

Severe complications of rotavirus infections are rare and pathogenesis remains unclear. Genotype variations of rotavirus, endotheliitis, cytokines such as interleukin 6 and 10 are emphasized. ${ }^{13}$ Also cellular immunodeficiency is associated with severity of rotavirus infection. ${ }^{14}$ In our patient, we did not identify the genotype of the virus. The patient may have an underlying immunodeficiency that we could not show with standard immunologic tests.

Acute cerebellitis associated with rotavirus has a distinct disease course with reduced consciousness and cerebellar mutism. Although rare, it should be considered especially in patients with a reversible splenial lesion because early and appropriate treatment may improve outcome. Dexamethasone treatment should be considered. Also rotavirus vaccination should be take into account to prevent severe complications. 


\section{REFERENCES}

1. Takanashi J, Miyamoto T, Ando N, et al. Clinical and radiological features of rotavirus cerebellitis. AJNR Am J Neuroradiol 2010; 31: 1591-1595.

2. Shiihara T, Watanabe I, Honma A, et al. Rotavirus associated encephalitis/encephalopathy and concurrent cerebellitis: report of two cases. Brain Dev 2007; 29: 670-673.

3. Raming RF. Pathogenesis of intestinal and systemic rotavirus infection. J Virol 2004; 78: 10213-10220.

4. Küper M, Timmann D. Cerebellar mutism. Brain Lang 2013; 127: 327-333.

5. Marien P, Verslegers L, Moens M, Dua G, Herregods $\mathrm{P}$, Verhoeven J. Posterior fossa syndrome after cerebellar stroke. Cerebellum 2013; 12: 686-691.

6. Pohl D, Alper G, Van Haren K, et al. Acute disseminated encephalomyelitis: updates on an inflammatory CNS syndrome. Neurology 2016; 87(9 Suppl 2): S38-S45.

7. Mori T, Morii M, Kuroiwa Y, Hotsubo T, Fuse S, Tsustumi H. Rotavirus encephalitis and cerebellitis with reversible magnetic resonance signal changes. Pediatr Int 2011; 53: 252-255.

8. Bosetti FM, Castagno E, Rainò E, Migliore G, Pagliero R, Urbino AF. Acute rotavirus-associated encephalopathy and cerebellitis. Minerva Pediatr 2016; 68: 387-378

9. Sheridan SL, Ware RS, Grimwood K, Lambert SB. Febrile seizures in the era of rotavirus vaccine. J Pediatric Infect Dis Soc 2016; 5: 206-209.

10. Orrico-Sánchez A, López-Lacort M, Muñoz-Quiles C, Díez-Domingo J. Lack of impact of rotavirus vaccines on seizure-related hospitalizations in children under 5 years old in Spain. Hum Vaccin Immunother 2018; 14: 1534-1538.
11. Hattori F, Kawamura Y, Kawada JI, et al; Aichi Pediatric Clinical Study Group. Survey of rotavirusassociated severe complications in Aichi Prefecture. Pediatr Int 2018; 60: 259-263.

12. Engan M, Bjørlykke JA, Moen G, Lund KB, Njølstad G. A 4-year-old girl with diarrhoea, paresis and mutism. Tidsskr Nor Laegeforen 2016; 136: 14581460.

13. Gotoh K, Nishimura N, Kawabe S, et al. Pathophysiological analysis of five severe cases with rotavirus infection. JMM Case Rep 2015; 2.

14. Rosenfeld L, Mas Marques A, Niendorf S, et al. Life-threatening systemic rotavirus infection after vaccination in severe combined immunodeficiency (SCID). Pediatr Allergy Immunol 2017; 28: 841-843.

15. Nigrovic LE, Lumeng C, Landrigan C, Chiang VW Rotavirus cerebellitis? Clin Infect Dis 2002; 34: 130.

16. Dickey M, Jamison L, Michaud L, Care M, Bernstein DI, Staat MA. Rotavirus meningoencephalitis in a previously healthy child and a review of the literature. Pediatr Infect Dis J 2009; 28: 318-321.

17. Kubota T, Suzuki T, Kitase Y, et al. Chronological diffusion-weighted imaging changes and mutism in the course of rotavirus-associated acute cerebellitis/ cerebellopathy concurrent with encephalitis/ encephalopathy. Brain Dev 2011; 33: 21-27.

18. Thompson MJ, Gowdie PJ, Kirkwood CD, Doherty RR, Fahey M. Rotavirus cerebellitis: new aspects to an old foe? Pediatr Neurol 2012; 46: 48-50.

19. Kato Z, Sasai H, Funato M, Asano T, Kondo N. Acute cerebellitis associated with rotavirus infection. World J Pediatr 2013; 9: 87-89. 